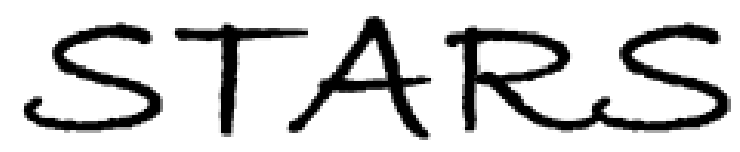

University of Central Florida

STARS

$1-1-2010$

\title{
Effective preemptive scheduling scheme for optical burst- switched networks with cascaded wavelength conversion consideration
}

Xingbo Gao

Mostafa A. Bassiouni

University of Central Florida

Guifang Li

University of Central Florida

Find similar works at: https://stars.library.ucf.edu/facultybib2010

University of Central Florida Libraries http://library.ucf.edu

This Article is brought to you for free and open access by the Faculty Bibliography at STARS. It has been accepted for inclusion in Faculty Bibliography 2010s by an authorized administrator of STARS. For more information, please contact STARS@ucf.edu.

\section{Recommended Citation}

Gao, Xingbo; Bassiouni, Mostafa A.; and Li, Guifang, "Effective preemptive scheduling scheme for optical burst-switched networks with cascaded wavelength conversion consideration" (2010). Faculty Bibliography 2010s. 7054.

https://stars.library.ucf.edu/facultybib2010/7054

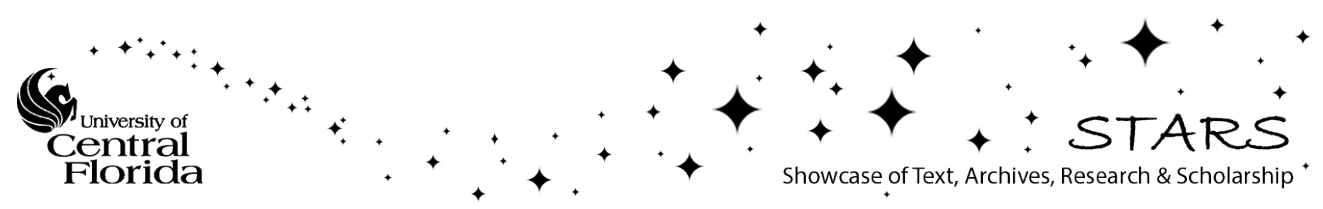




\section{Effective preemptive scheduling scheme for optical burst-switched networks with cascaded wavelength conversion consideration}

\author{
Xingbo Gao, MEMBER SPIE \\ Mostafa A. Bassiouni \\ University of Central Florida \\ School of Electrical Engineering and Computer \\ Science \\ 4000 Central Florida Boulevard \\ Orlando, Florida 32816 \\ E-mail: xbgao@cs.ucf.edu
}

Guifang Li, FELLOW SPIE

University of Central Florida

College of Optics

4000 Central Florida Boulevard

Orlando, Florida 32816

\begin{abstract}
We introduce a new preemptive scheduling technique for next-generation optical burst switching (OBS) networks considering the impact of cascaded wavelength conversions. It has been shown that when optical bursts are transmitted all optically from source to destination, each wavelength conversion performed along the lightpath may cause certain signal-to-noise deterioration. If the distortion of the signal quality becomes significant enough, the receiver would not be able to recover the original data. Accordingly, subject to this practical impediment, we improve a recently proposed fair channel scheduling algorithm to deal with the fairness problem and aim at burst loss reduction simultaneously in OBS environments. In our scheme, the dynamic priority associated with each burst is based on a constraint threshold and the number of already conducted wavelength conversions among other factors for this burst. When contention occurs, a new arriving superior burst may preempt another scheduled one according to their priorities. Extensive simulation results have shown that the proposed scheme further improves fairness and achieves burst loss reduction as well. () 2010 Society of Photo-Optical Instrumentation Engineers. [DOI: 10.1117/1.3363610]
\end{abstract}

Subject terms: optical burst switching; wavelength-division multiplexing; preemption; fairness.

Paper 090835PR received Oct. 28, 2009; revised manuscript received Jan. 18, 2010; accepted for publication Jan. 19, 2010; published online Mar. 29, 2010.

\section{Introduction}

It is well-known that optical transmission is playing a critical role in the backbone of commercial telecommunication networks. Nowadays, a single-wavelength channel can transmit data at a rate of $10 \mathrm{~Gb} / \mathrm{s}$ and beyond under wavelength-division multiplexing (WDM). Dense WDM (DWDM) technology allows tens or even hundreds of wavelength channels to be transmitted over a single optical fiber. Properly designed and operated, optical switching technologies can potentially utilize this immense bandwidth in order to meet the growing enormous demand from the Internet. Optical circuit switching has been available, but it offers only a coarse granularity of switching. On the other hand, optical packet switching will not be favorable in the foreseeable future until optical buffers outgrow their immaturity, even though it can switch at the packet level with fine granularity. Optical burst switching (OBS) 13 emerges as a viable technology by consolidating the currently available techniques.

OBS is a promising bufferless DWDM switching technology that can potentially provide high wavelength utilization. In OBS networks, data packets are aggregated into much larger sized bursts before transmission. A data burst is preceded in time by a control packet, which is sent on a separate control wavelength and requests resource allocation at switches. Bursts are typically released into the optical layer before the acknowledgment of a successful light-

0091-3286/2010/\$25.00 @ 2010 SPIE path reservation. Several such one-way reservation protocols have been proposed, and the just-enough-time (JET) reservation scheme, which reserves the bandwidth on a control channel only for the duration of the bursts, has received the most attention.

In OBS networks, burst contention may arise at any intermediate node during channel scheduling. Indeed, when a control packet arrives at a node to make the wavelength reservation for the associated incoming burst, it may happen that the requested resource is unavailable, as it is occupied by another burst. Unlike optical circuit switching, it will incur too much overhead in OBS if we try to develop a wavelength scheduling scheme on the basis of global link state exchanging in order to avoid or reduce burst contention. This is mainly because bursts are relatively short and link states change too frequently. Therefore, investigations of burst loss performance in OBS often assume that contention resolution is achieved in the yavelength domain through full wavelength conversion 246 In full wavelength conversion, every optical cross-connect (OXC) is equipped with full-range wavelength converters that can convert an incoming wavelength to any of the outgoing wavelengths. However, it is well understood that wavelength conversion degrades the quality of the signal and reduces the signal-tonoise ratio (SNR); cascaded wavelength conversions further aggravate this problem and thus must be carefully handled?

To provide accurate and better guidelines for the optical switching community, we need to consider the practical impediments and constraints that challenge the deployment 
of the current wavelength conversion technology when conducting performance evaluation. Taking the accumulative negative impact of cascaded wavelength conversions into account, the use of wavelength converters in OBS networks is hence constrained by a bound on the number of wavelength conversions that a signal can go through all optically. We have named this constrain conversion cascading constraint in our previous paper We also showed that the conversion cascading constraint may inevitably cause noticeable or even significant negative impact on the burst loss performance in OBS with full wavelength conversion. More seriously, it is common to most networks that the longer the hop route a transmission has to traverse, the greater the risk of being blocked. In OBS networks, this is the fairness problem in terms of loss rate among data bursts with various hop counts. Since bursts with longer hops may experience more wavelength conversions during transmission, the unfairness may also deteriorate under the effect of the conversion cascading constraint. In this paper, we propose a new preemption-based scheduling scheme, which also manages to reduce the converter usage, to resolve the fairness problem. The proposed scheme adapts the fair channel scheduling algorithm, recently introduced by Hsu and Yang 10 to the environment of cascaded wavelength conversions bounded by a threshold. The extensive simulation results demonstrate that our scheme not only can alleviate the aggravated unfairness defect of popular signaling protocols such as JET, but also can improve burst loss performance to a certain extent.

The rest of this paper is organized as follows; Section 2 discusses related work about designing channel reservation algorithms to improve burst loss performance and fairness as well in OBS networks. Section 3 describes the proposed conversion reduction and fair prioritized preemption scheduling scheme. Numerical results are demonstrated in Sec. 4. Last, Sec. 5 concludes the paper.

\section{Related Work}

In this section, we first discuss the fundamental wavelength scheduling algorithms and the contention problem, and then we investigate the fairness problem and several relevant approaches addressed in previous literature.

In JET-based OBS, data bursts are assigned variable offset lengths at edge nodes according to their path distance, and as the bursts are traveling through the network, these offsets will shrink. The presence of this variability and the dynamic random arrival of bursts create a large number of idle periods (voids) on wavelength channels. The scheduler in each optical node faces herein the challenge that it must accommodate efficiently bursts in the absence of global link state information. To overcome this difficulty, Xiong et al 4 proposed two scheduling algorithms called latest available unscheduled channel (LAUC) and LAUC with void filling (LAUC-VF), respectively. LAUC, which is the same as the horizon algorithm, maintains a single variable recording the latest reservation time of each channel and assigns the channel with the latest starting time that is still earlier than the arrival time of the incoming burst. LAUC is simple but cannot utilize all existing voids. LAUC-VF keeps track of all void intervals within the channel space and assigns the intervals that would give the minimum of gaps or voids. This has the effect of filling channel space more effectively, ensuring that any newly created voids would occur closer to the present time and hence be more capable of being filled by newly arriving bursts. The minimum starting void (Min-SV) algorithm 11212 uses a geometric approach and organizes the voids into a balanced binary tree. The Min-SV algorithm finds a void that minimizes the distance between the starting time of the void and the starting time of the burst. It can produce burst schedules as efficient as LAUC-VF but more quickly. A more efficient scheduling approach proposed by Chen et al 13 that requires a special hardware-based constant time burst resequencing (CTBR) scheduler can achieve only $\mathrm{O}(1)$ runtime complexity. CTBR is similar to the free channel queue (FCQ) burst scheduling algorithm, 1415 which fits in with the new dualheader optical burst switching (DOBS) architecture.

One of the primary objectives in the design of an OBS network is to minimize burst loss. Burst loss occurs primarily due to the contention of bursts in the bufferless core nodes. During scheduling, an arriving burst may contend with one or more scheduled bursts on the outgoing data channels. This contention results in the burst being dropped, leading to burst loss. Approaches for resolving contention include wavelength conversion $16 / 17$ optical

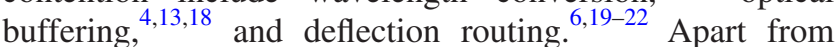
these three contention resolution approaches, burst segmentation 23.24 and preemption technique $25 \sqrt{27}$ were also proposed.

In OBS networks, the fairness problem causes the loss probabilities of optical bursts traveling through lightpaths with larger hop counts to be higher than those whose paths have a smaller number of hops. This is another important topic that we need to face and solve. The fairness problem was investigated by Wang et al 6 as a secondary consideration during the evaluation of the deflection routing algorithm. But the authors showed that their proposed deflection routing could neither improve nor aggravate unfairness for various kinds of bursts. The work 102830 were dedicated to improving fairness in OBS networks. The monitoring group drop probability (MGDP) approach 28 intentionally drops a burst with a small total hop count so that more resources can be left for bursts with longer paths. However, this feature may cause unnecessary burst drops, and consequently, blocking performance is sacrificed to satisfy fairness. Another disadvantage of MGDP is that it focuses on achieving fairness on a single switching node but may worsen the fairness of bursts transmission in the whole network as a result. The balanced just-in-time scheme (BJIT) 29 deals with the fairness problem by adjusting the size of the search space for a free wavelength based on the number of hops traveled by the burst. The size for searching is designed to grow gradually as the burst approaches to its destination. Similar to MGDP, BJIT may suffer from higher burst losses because some bursts with a short lightpath have difficulty in finding free wavelengths at their first or even second hop and get dropped excessively. Recently, we proposed a suite of three-hop-based fairness-improving adaptive routing schemes 31 The new approaches consider the transient link congestion at the moment when the bursts arrive and use this information to reduce the overall burst 
Table 1 Negative impact of the conversion cascading constraint on fairness (U.S. LongHaul, load $=0.06$ ).

\begin{tabular}{cccccccc}
\hline \hline \multirow{2}{*}{ Constraint } & One-hop & Two-hop & Three-hop & Four-hop & Five-hop & Six-hop & Seven-hop \\
\hline$n c$ & $6.19 \mathrm{E}-4$ & 0.001631 & 0.003054 & 0.004532 & 0.006056 & 0.007606 & 0.00704 \\
$c_{3}$ & $6.14 \mathrm{E}-4$ & 0.001614 & 0.003036 & 0.004501 & 0.029045 & 0.072899 & 0.107434 \\
$c_{2}$ & $5.69 \mathrm{E}-4$ & 0.001491 & 0.00281 & 0.052729 & 0.126291 & 0.200644 & 0.237704 \\
$c_{1}$ & $3.42 \mathrm{E}-4$ & $8.62 \mathrm{E}-4$ & 0.133151 & 0.268722 & 0.393779 & 0.496653 & 0.537703 \\
\hline \hline
\end{tabular}

loss probability. The proposed schemes also resolve the intrinsic unfairness defect of existing popular signaling protocols.

In addition to providing contention solution and achieving service differentiation, as shown in past works, 25 preemption has also been used to offer fair scheduling in JETbased OBS networks as well. This is accomplished by carefully designing the preemption scheme so that the bursts to be discarded are selected in such a way that fairness is improved without causing significant deterioration in network performance. The authors 30 proposed to use constrained preemption to improve fairness without degrading network throughput. They set a couple of additional constraints to reduce resource waste and improve efficiency of preemption. On the other hand, Hsu and Yang 10 first provided an in-depth analysis of the fairness problem in JET-based OBS networks. They then derived a priority function evaluated on a group of parameters inferred from their analysis: successful hops, remaining hops, initial offset time, and average burst duration time. Preemption (taking account of both fairness and loss performance) is triggered based on the priority function in case of any burst contention. The resulting fair prioritized preemption (FPP) algorithm is remarkable, and the simulation results showed that it yields better fairness and lower losses than the other two fairness solutions-MGDP and BJIT. Therefore, our proposed preemptive scheduling scheme is based on FPP. To accommodate the conversion cascading constraint that the current converter technology is facing, we reduce the converter usage whenever possible and combine a constraint threshold into a new priority function. The details of the algorithm are presented in the following section. The techniques introduced by Zhou et al 30 could be incorporated into our preemption solution for further improvement, and we may consider them in real implementation.

\section{Conversion Reduction and Fair Prioritized Preemption}

In this section, we describe the proposed wavelength scheduling scheme-conversion reduction and fair prioritized preemption algorithm (CR-FPP)—in detail. The algorithm illustration is preceded with an example exhibiting the essentiality of special treatment of the conversion cascading constraint to obtain fairness.

It is intuitive to infer that the burst loss performance will be degraded when the conversion cascading constraint is in effect. This is because some bursts that can get transmitted successfully through uncapped conversions may be dis- carded due only to this constraint. On the other hand, we show here that this constraint would probably worsen the fairness issue too.

Table 1 shows the relationship between loss rate and hop count with various thresholds of the conyersion cascading constraint in the U.S. LongHaul network? The number of wavelengths per fiber link $(W)$ is equal to six, $c_{i}$ denotes the performance obtained when the maximum allowed number of cascaded conversions is $i$, while $n c$ indicates the performance obtained when the cascading constraint does not exist. In other words, when $c_{i}$ is in effect, if any burst requires more than $i$ cascaded wavelength conversions for its endto-end transmission, it will be dropped at the last intermediate node. JET is used as the signaling protocol in this simulation. The performance results for $n c$ act as the baseline during comparison. It is clear from Table 1 that even under no existence of the conversion cascading constraint, the unfairness among bursts of different hop counts is still significant. For example, seven-hop bursts have 10 times the loss rate of one-hop bursts (0.00704 versus 6.19E-4). We thus can reason that the fairness problem would worsen if we take the cascading constraint into account. Since $i$-hop bursts require a maximum of $i-1$ wavelength conversions for successful transmission, the loss rate of bursts with four or fewer hops is not expected to increase because of the presence of constraint $c_{3}$. Similarly, bursts with three hops or fewer are not affected by the constraint $c_{2}$, and so on. Actually, we observe in Table 1 that under $c_{3}$, the loss performance of one-hop to four-hop bursts has improved to different extents compared with the case of $n c$, but the losses of longer-hop bursts have increased adversely, which makes the fairness problem much more serious. A similar phenomenon happens for $c_{2}$ and $c_{1}$. This behavior is understandable because when longer-hop bursts are excessively blocked due to the cascading constraint, more wavelengths are left free for shorter-hop bursts.

We use the following same notations as in FPP (Ref. 10 to present CR-FPP:

- $X_{i}$ : any new burst arriving at an optical node along its lightpath

- $H_{i}$ : total hop counts of burst $X_{i}$ for its end-to-end transmission

- $\sigma_{i}$ : successful hop counts that burst $X_{i}$ has traversed so far

- $\tau_{i}$ : remaining hop counts of burst $X_{i}$, i.e., $H_{i}=\sigma_{i}+\tau_{i}$

- $L$ : average burst duration time

- $\delta$ : processing time of a control packet at core nodes 


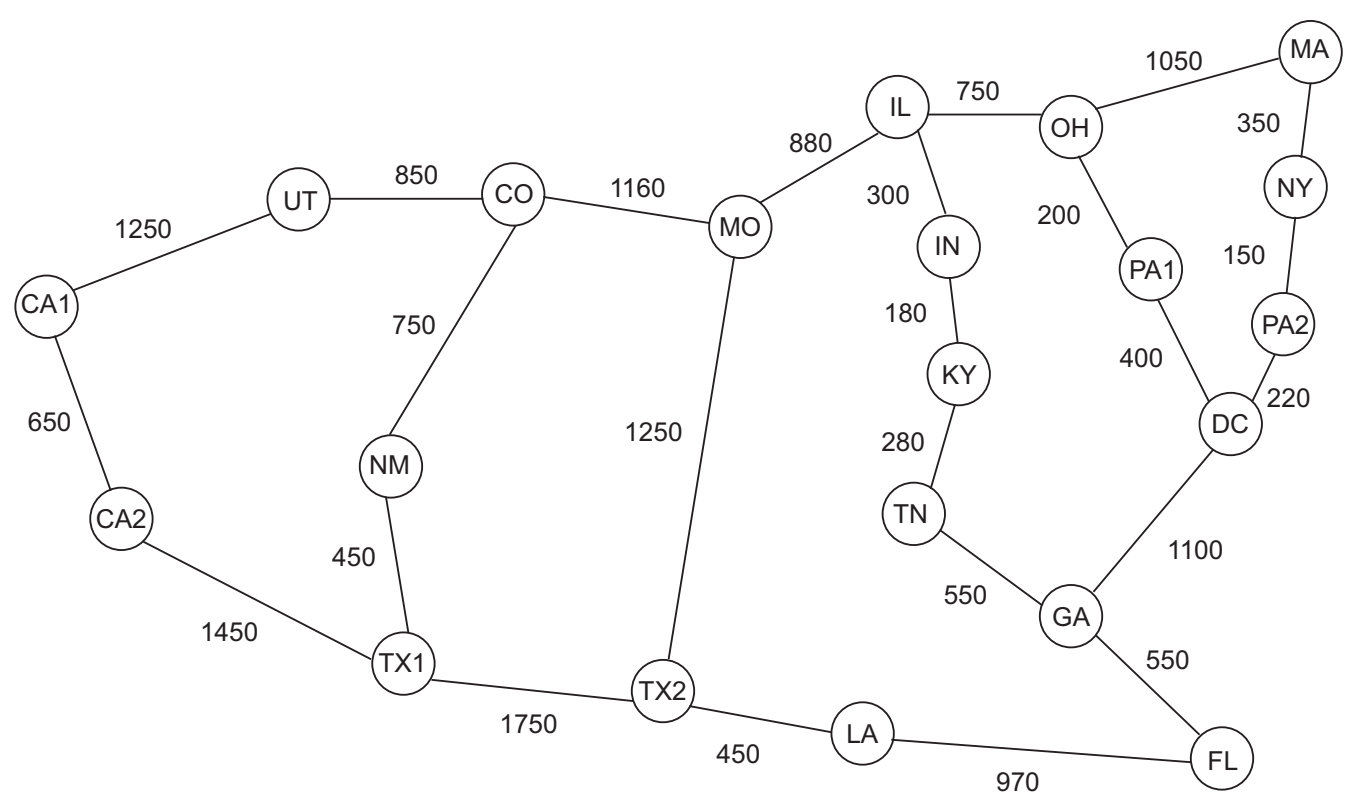

Fig. 1 21-node Abilene (Internet2) network with link distance in km.

- $\Delta_{i}$ : initial offset time of burst $X_{i}$, i.e., $\Delta_{i}=H_{i} \times \delta$

- $\theta$ : the evaluation function for preemption

As pointed out by Hsu and Yang, 10 the total number of hops pertains to fairness, the remaining hops can promote the priority of bursts close to its destination, and the number of successful hops is relevant to link utilization. FPP integrates these considerations into the evaluation function $\theta$ to achieve fairness improvement and efficient link utilization together. The evaluation function $\theta$ biased to longer $H_{i}$ at some point is given as Eq. (1)

$\theta\left(X_{i}\right)=\sigma_{i}-\left(\Delta_{i} / L\right) \times \tau_{i}$.

In Eq. (1) the negative term is used to prune the biased preference for bursts with longer initial offset time, and the significance is determined by the ratio $\Delta_{i} / L$. If this pruning is not executed, the preceeding preemptive scheme may apparently overcorrect the fairness problem.

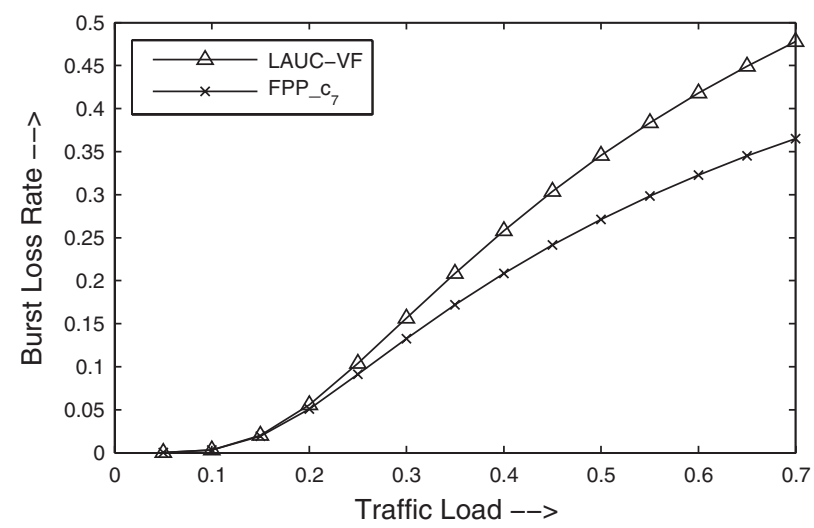

Fig. 2 Burst loss: pure LAUC-VF versus FPP $\left(c_{\max }=7\right)$.
To address the negative performance impact caused by the conversion cascading constraint, we propose algorithm CR-FPP and show its pseudocode as follows:

Input: Burst $X_{\text {new }}=\left\langle H_{\text {new }}, \sigma_{\text {new }}, \tau_{\text {new }}, \Delta_{\text {new }}, \pi_{\text {new }}, \lambda_{\text {prev }}\right\rangle, c_{\text {max }}$, and the current schedule

Output: Grant reservation request of $X_{\text {new }}$ or not

if $X_{\text {new }}$ can be scheduled into the current schedule on $\lambda_{\text {prev }}$ then

Reserve $X_{\text {new }}$ on $\lambda_{\text {prev }}$; return;

else if $X_{\text {new }}$ can be scheduled on $\lambda_{m}\left(\neq \lambda_{\text {prev }}\right)$ via LAUC-VF then

Reserve $X_{\text {new }}$ on $\lambda_{m}$; return;

else

$\Psi \leftarrow \phi ;$

for $i=1$ to $W$ do

$X_{\text {con }}=\left\langle H_{c o n}, \rho_{c o n}, \tau_{c o n}, \Delta_{c o n}, \pi_{c o n}\right\rangle \quad /^{*}$ the contending burst on $\lambda_{i}{ }^{*}$

if $\bar{\theta}\left(X_{\text {new }}\right)>\bar{\theta}\left(X_{\text {con }}\right)$ then

$\Psi \leftarrow \Psi \cup\left\{X_{c o n}\right\} ;$

end if

end for

if $\Psi \neq \phi$ then

Pick $X_{j}$ such that $\left(\sigma_{j} / H_{j}\right)=\min _{\forall X_{i}}\left(\sigma_{i} / H_{j}\right)$

Reserve $X_{\text {new }}$ on the channel that $X_{j}$ was scheduled;

Send message to release reservations for $X_{j}$ on both uplink(s) and downlink(s);

else

Drop $X_{\text {new; }}$

end if

return;

end if

CR-FPP first tries to schedule the incoming burst on its 


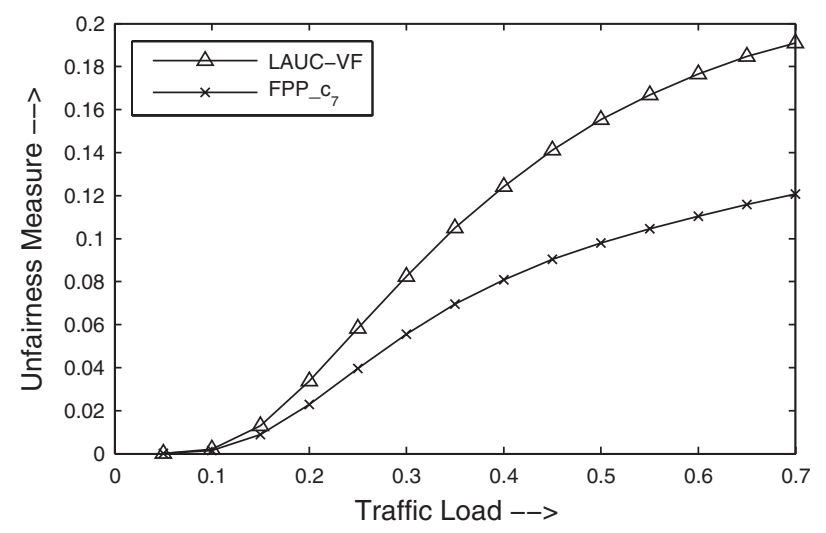

Fig. 3 Unfairness measure: pure LAUC-VF versus FPP $\left(c_{\max }=7\right)$.

current channel $\lambda_{\text {prev }}$ that it used at the previous hop(s). Only if the channel occupied by the incoming burst is not available on the outgoing link is LAUC-VF scheduling applied. If LAUC-VF still cannot accommodate the new burst, CR-FPP will look for the candidate bursts to preempt according to the new evaluation function $\bar{\theta}$ :

$\bar{\theta}\left(X_{i}\right)=\left(1-\frac{\pi_{i}}{c_{\max }} \cdot \frac{1}{\sqrt{H_{i}}}\right) \times \sigma_{i}-\beta \times\left(\Delta_{i} / L\right) \times \tau_{i}$,

where $\pi_{i}$ denotes the accumulated number of wavelength conversions conducted for burst $X_{i}$ so far, $c_{\max }$ is the threshold, and $\pi_{i} \leqslant c_{\max }$. Due to the cascading constraint, we determine the eligibility of a candidate burst not only by how many successful hops $\left(\sigma_{i}\right)$ it has traversed, but also by how many wavelength conversions $\left(\pi_{i}\right)$ it has experienced. The term $\left(\pi_{i} / c_{\max }\right) \cdot\left(1 / \sqrt{H_{i}}\right)$ represents the latter impactor and should impose a negative impact. It is in inverse proportion to $H_{i}$, which gives more bias on longer-path bursts to achieve fairness, because as mentioned earlier, those bursts may be affected by the constraint more often in general. In Eq. (2) a new parameter $\beta$ is also added to the second term relevant to $\tau_{i}$. This parameter $\beta$ provides us with more flexibility determining the significance of $\tau_{i}$. We can fine-tune $\beta$ to further reduce the two opposite side effects brought by the preemption scheme: overcorrection because of the overpreference for bursts with longer initial offset time and oversuppression of longer-hop bursts due to possible increasing packet process time $\delta$. We will discuss the effects in more detail in Sec. 4. When a burst is preempted, messages are sent to both uplink(s) and downlink(s) to release the resources reserved for this burst.

Similar to FPP, in algorithm CR-FPP, if more than one scheduled burst loses in a contention, the algorithm picks the one with the shortest relative successful path as the victim to be preempted in this contention, i.e., burst $X_{j}$ such that $\left(\sigma_{j} / H_{j}\right)=\min _{\forall X_{i}}\left(\sigma_{i} / H_{i}\right)$. If two or more bursts on a channel are involved in a contention-in other words, over one scheduled burst shall be preempted to accommodate the single new burst-we discard the new burst directly to save the possible bandwidth waste.

The two channel scheduling schemes share similar time complexity. The basic LAUC-VF takes $O(M)$ time to schedule a burst, where $M$ is the number of voids per channel. If LAUC-VF fails, both FPP and CR-FPP shall scan each wavelength channel for any applicable contending bursts that may be preempted based on the evaluation function $\theta / \bar{\theta}$. Let $\bar{M}$ denote the average number of voids for all $W$ channels, then the two schemes both take $O(\bar{M} W+W)$ time for scheduling. However, CR-FPP involves more overheads in dealing with the cascading constraint, such as recording the channel used in previous hops as well as the number of cascaded wavelength conversions performed so far and utilizing a more complex preemption evaluation function.

\section{Numerical Results}

In this section, we investigate the burst loss performance and the fairness of the proposed preemptive scheduling scheme. Since it has been shown that FPP (Ref. 10 outperforms both BJIT and MGDP, we will focus on comparing CR-FPP with FPP only. The evaluation network model is the topology comprising the optical routing nodes extracted from the Abilene (Internet2) network. In the simplified Abilene topology (Fig. 1), the longest-shortest path between source and destination has seven intermediate OXCs. Therefore, no burst needs more than seven wavelength con-

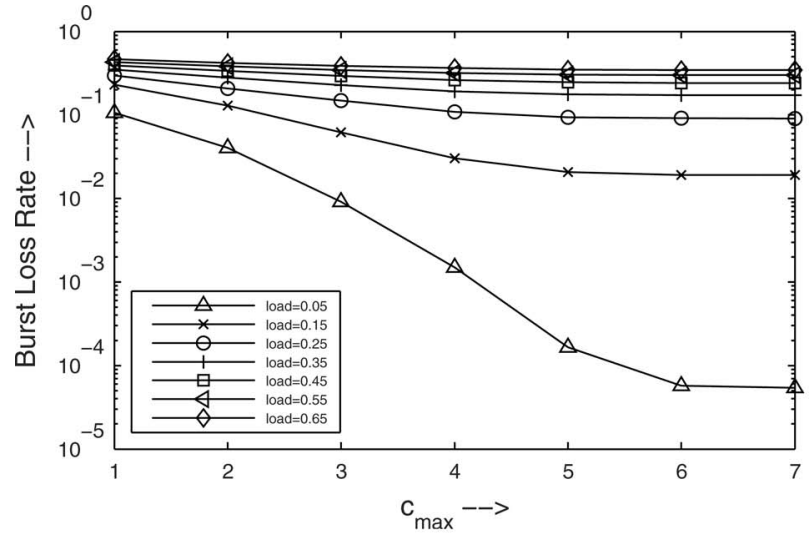

(a)

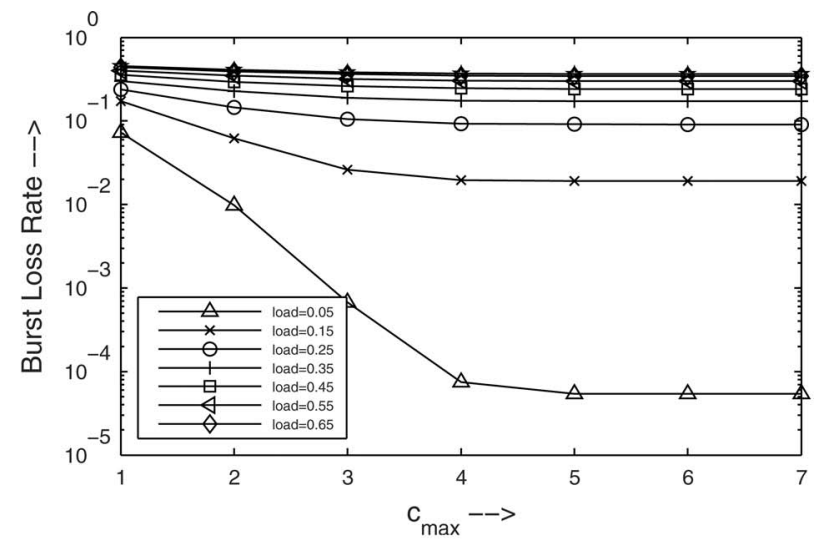

(b)

Fig. 4 Burst loss versus $c_{\max }$ (a) FPP. (b) CR-FPP. 


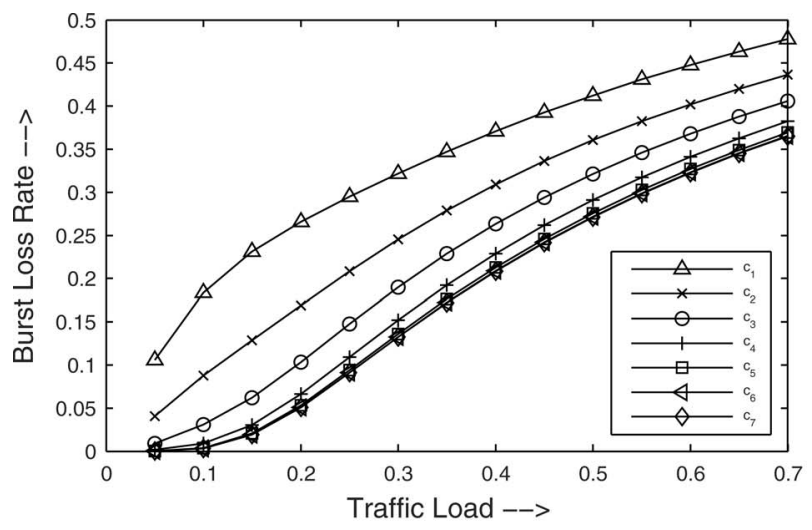

(a)

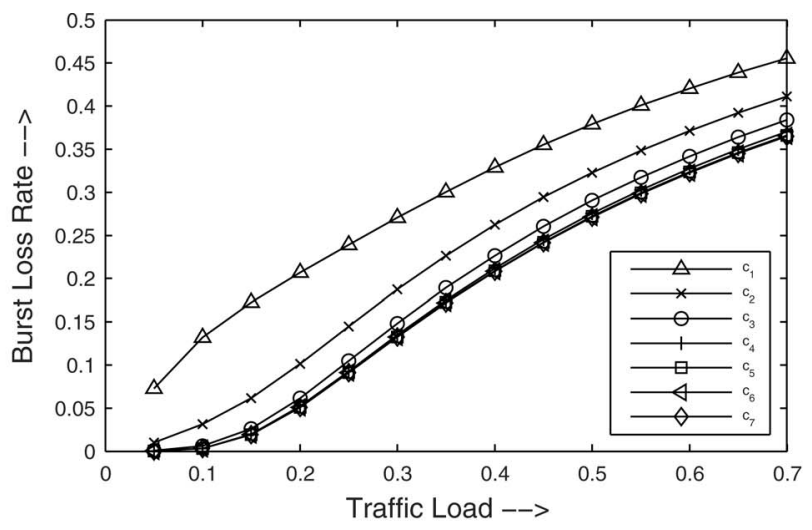

(b)

Fig. 5 Burst loss versus traffic load. (a) FPP. (b) CR-FPP.

versions to reach its destination. We assume that all nodes have the capability of full-range wavelength conversion. Every link is a bidirectional fiber and consists of $W$ data channels. The burst arrival pattern follows a Poisson process, and the burst duration time is negatively exponentially distributed with mean $L$. Traffic load is normalized with $\rho$ $=(\lambda L) / W$ per optical node. For each burst arrival, the source and destination nodes are uniformly selected. Unless specified, $W=8$, the ratio $\delta / L=0.01$, and $\beta$ in CR-FPP is set to 1.0. The unfairness measure is defined as the standard deviation of mean dropping probabilities calculated according to statistics of bursts with identical total hop count. In Fig. 1] the longest-shortest path has eight hops in total, and if we use $p_{i}$ to indicate the burst loss probability for $i$-hop bursts, where $1 \leqslant i \leqslant 8$, then the unfairness measure is calculated as the square root of their variance (based on their mean). The lower the unfairness measure is, the fairer a scheduling algorithm is. Each data point shown in the performance graphs is obtained by running $10^{7}$ burst transmission requests.

We first briefly illustrate the advantages of FPP over the pure LAUC-VF scheduling algorithm under no effect of the conversion cascading constraint. Figures 2 and 3 plot the burst loss rate and unfairness measure against traffic load, respectively. When $c_{\max }=7$, FPP will not be affected by the

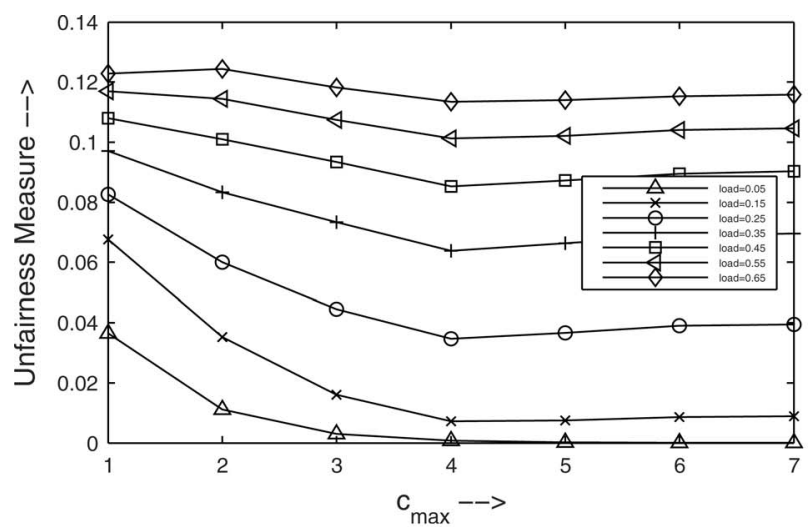

(a) cascading constraint at all. Because of its intelligent discarding, FPP shows large performance improvement for both burst loss and fairness. In short, FPP would not drop any burst deliberately, and it determines burst priorities dynamically for preemption.

Figures 46 compare the performance achievements between CR-FPP and FPP side by side. We have the following observations:

- CR-FPP achieves lower burst loss rates across almost all kinds of loads, and regardless of the value of $c_{\max }$. Moreover, as depicted in Figs. 4(a) and 4(b) for the same load, CR-FPP is able to suppress the negative impact of the conversion cascading constraint much faster than FPP when this constraint becomes less stringent (with higher $c_{\max }$ ), especially under lower loads. For instance, under load 0.05, CR-FPP has reached the stable loss rate at $c_{4}$, but FPP still suffers from high burst losses at the same $c_{4}$, which is over one order of magnitude in disparity. The merit of CRFPP lies in two aspects: at first, it effectively reduces unnecessary wavelength conversions by scheduling the bursts on the channel that they have used at their previous hop(s); second, it incorporates the consideration of the cascading constraint into the priority

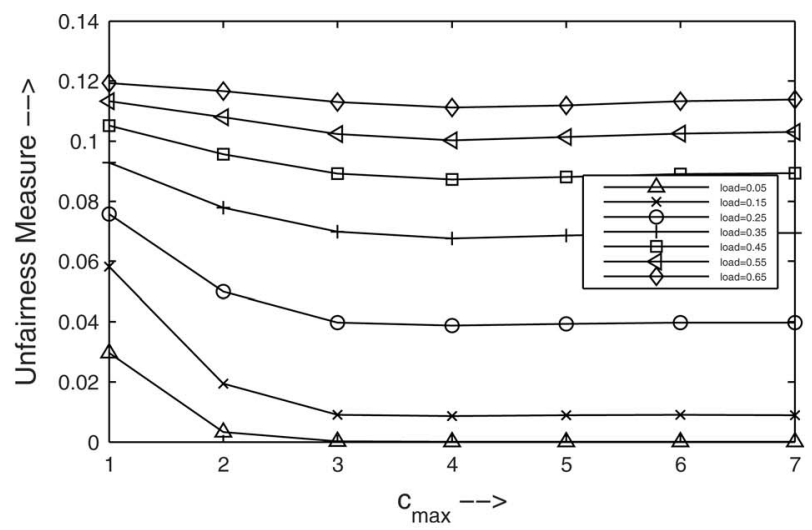

(b)

Fig. 6 Unfairness measure versus $c_{\max }$ (a) FPP. (b) CR-FPP. 


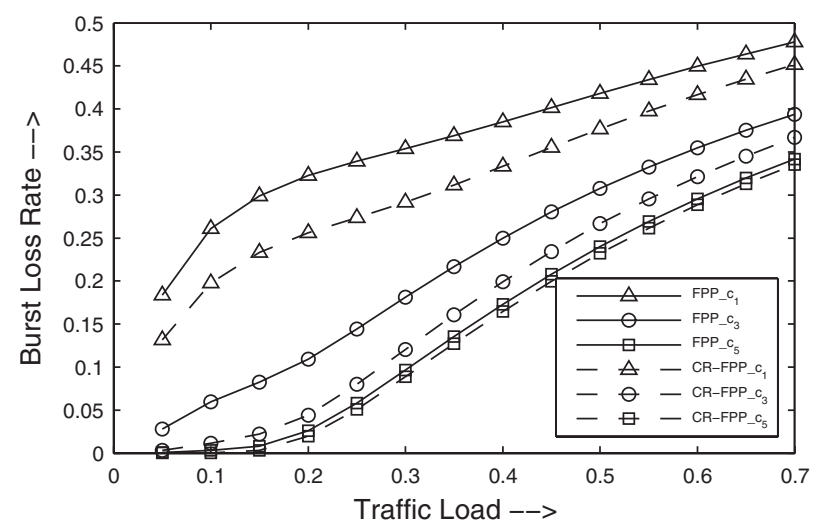

Fig. 7 Burst loss versus traffic load at $W=16$.

evaluation function for preemption. In this way, some bursts that have experienced relatively too many wavelength conversions, which are very possible to get dropped at their next hop(s) due to the cascading constraint, may be sacrificed at the current hop to accommodate other bursts with fewer conversions. Consequently, resource wastes have been saved ahead of time.

- Figures 5(a) and 5(b) further prove the preceding findings. It is clear that starting at $c_{4}$, CR-FPP almost obtains the same loss performance as when the cascading constraint is not in effect (i.e., $c_{\max } \geqslant 7$ ). The difference between the loss rates under the constraints $c_{\text {max }} \leqslant 3$ and the loss rates under the constraint $c_{7}$ is much smaller when using CR-FPP [Fig. 5(b)] than when using FPP [Fig. 5(a)].

- Owing to the similar reasons explained earlier, Figs. 6(a) and 6(b) show that CR-FPP achieves better fairness too for the same load.

Figures 7 and 8 depict the performance comparisons between CR-FPP and FPP for $c_{1}, c_{3}$ and $c_{5}$ at $W=16$. CR-FPP is superior, as expected. On the other hand, it is noticeable that for either algorithm, the performance disparity between $c_{1}$ and $c_{3}$ or between $c_{3}$ and $c_{5}$ (e.g., CR-FPP $c_{1}$ versus CR-FPP_c $c_{3}$ ) is bigger than that when $W=8$, especially at lower loads. This can be interpreted as follows. Both algorithms are expected to use LAUC-VF for scheduling most

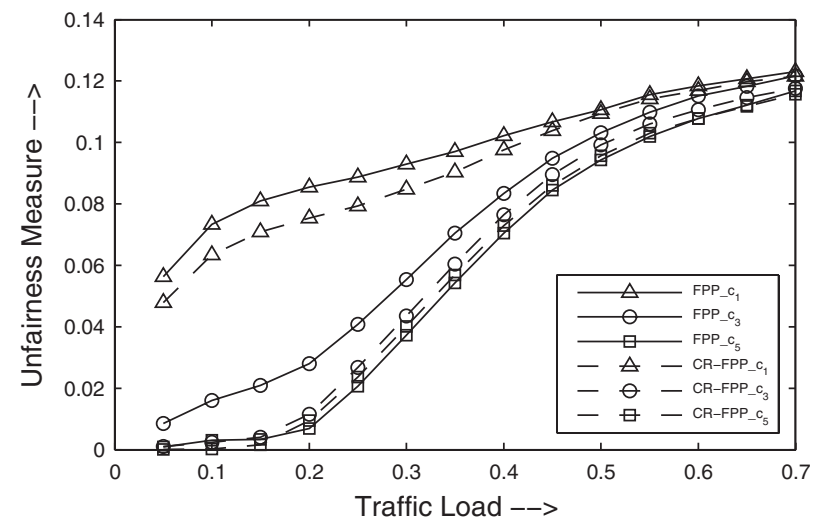

Fig. 8 Unfairness measure versus traffic load at $W=16$.

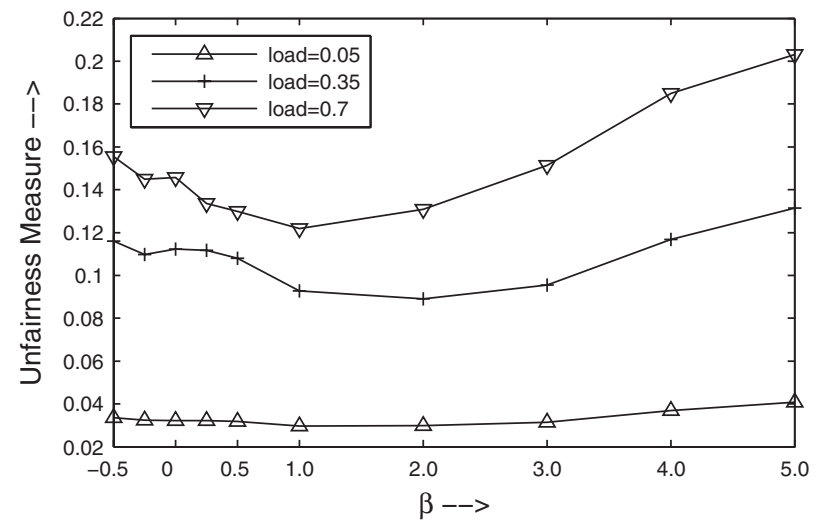

Fig. 9 Unfairness measure versus $\beta$ under $c_{1}$.

of the time. Actually, pure LAUC-VF is a kind of channel scheduling algorithm that is biased to converter utilization. It performs exhaustive searching, trying to find the matching void throughout the available ones on all channels. When $W$ gets larger, there will be more channels as well as more voids available, and consequently, more conversions would occur. Generally speaking, the larger the number of conversions, the more severe the negative impact of the cascading constraint would be, whereas since CR-FPP possesses the intelligence to deal with the cascading constraint, it is still able to maintain a very small performance discrepancy between CR-FPP_c $c_{3}$ and CR-FPP_ $c_{5}$.

We also exploit the impact on the network performance when the parameter $\beta$ varies. In the original FPP algorithm, the term $\left(\Delta_{i} / L\right) \times \tau_{i}$ was designed to suppress the overpreference for bursts with longer initial offset time. However, we expect this significance would also be related to a variety of other conditions such as network topology, traffic load, and the cascading constraint threshold in our studying case. Tables 2 and 3 display burst loss variations when $\beta$ grows under $c_{1}$ and $c_{5}$, respectively, while Figs. 9 and 10 show fairness fluctuations. To ensure completeness, we consider some negative $\beta$ values too. We can observe that burst loss rates have been decreasing when $\beta$ increases for the values of $\beta$ we select. On the contrary, fairness has shown a different curve roughly decreasing at first, reach-

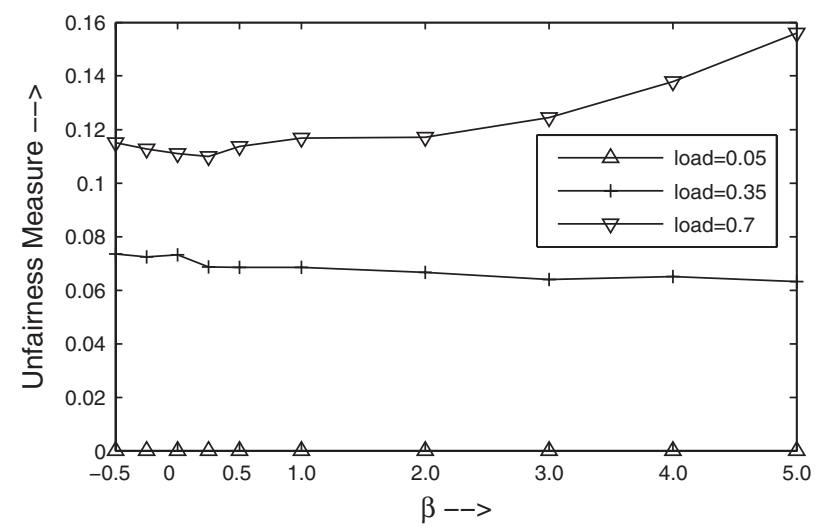

Fig. 10 Unfairness measure versus $\beta$ under $c_{5}$. 
Table 2 Impact of $\beta$ on burst loss under $c_{1}$.

\begin{tabular}{|c|c|c|c|c|c|c|c|c|c|c|}
\hline $\mathrm{Load} / \beta$ & -0.5 & -0.25 & 0.0 & 0.25 & 0.5 & 1.0 & 2.0 & 3.0 & 4.0 & 5.0 \\
\hline 0.05 & 0.072718 & 0.072696 & 0.072671 & 0.072684 & 0.072681 & 0.072513 & 0.072023 & 0.071857 & 0.071645 & 0.07155 \\
\hline 0.35 & 0.307824 & 0.307779 & 0.306885 & 0.302778 & 0.302713 & 0.300531 & 0.295888 & 0.293615 & 0.290728 & 0.289678 \\
\hline 0.70 & 0.483667 & 0.483264 & 0.478419 & 0.458995 & 0.458594 & 0.455494 & 0.448272 & 0.442965 & 0.437204 & 0.43502 \\
\hline
\end{tabular}

Table 3 Impact of $\beta$ on burst loss under $c_{5}$.

\begin{tabular}{|c|c|c|c|c|c|c|c|c|c|c|}
\hline $\mathrm{Load} / \beta$ & -0.5 & -0.25 & 0.0 & 0.25 & 0.5 & 1.0 & 2.0 & 3.0 & 4.0 & 5.0 \\
\hline 0.05 & 0.000054 & 0.000054 & 0.000054 & 0.000054 & 0.000054 & 0.000054 & 0.000054 & 0.000054 & 0.000054 & 0.000054 \\
\hline 0.35 & 0.181062 & 0.180544 & 0.179496 & 0.173313 & 0.172721 & 0.172348 & 0.172114 & 0.171344 & 0.170118 & 0.169275 \\
\hline 0.70 & 0.399438 & 0.398218 & 0.393653 & 0.368539 & 0.366999 & 0.366195 & 0.365374 & 0.362781 & 0.359315 & 0.357135 \\
\hline
\end{tabular}

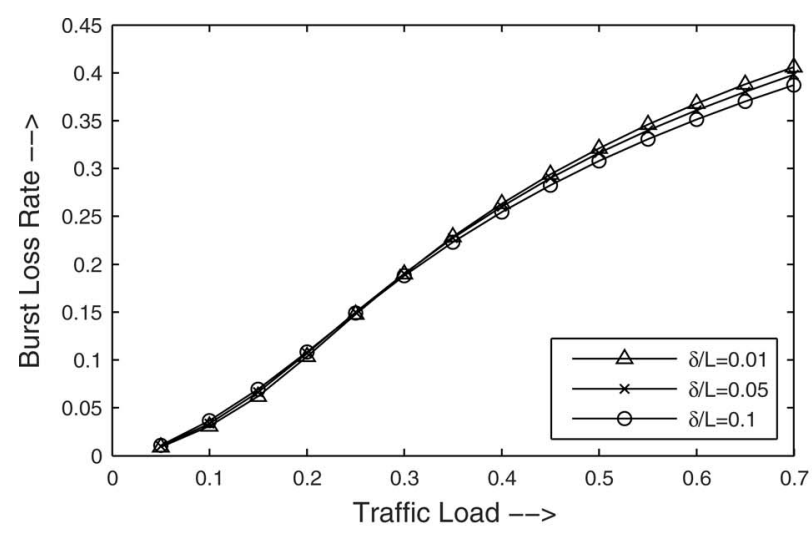

(a)

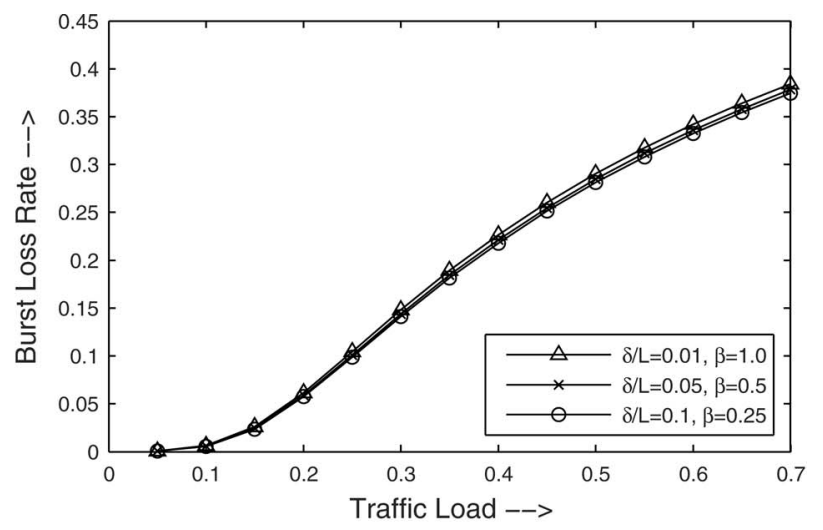

(b)

Fig. 11 Burst loss versus traffic load. (a) FPP. (b) CR-FPP.

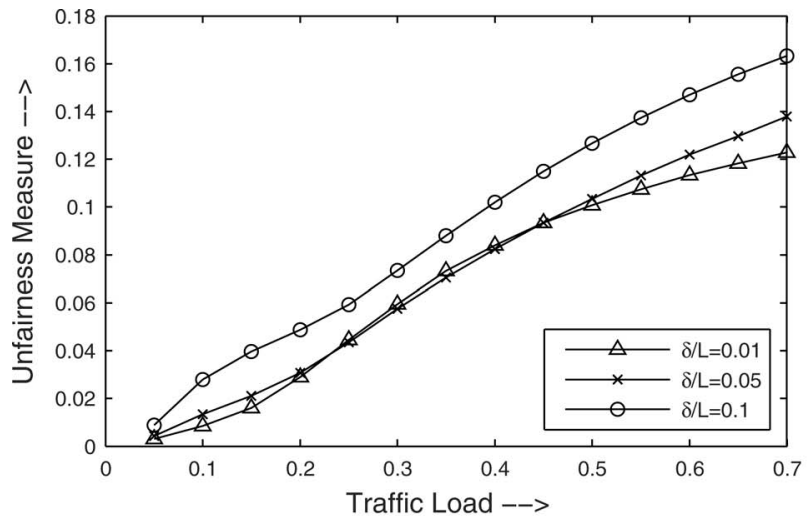

(a)

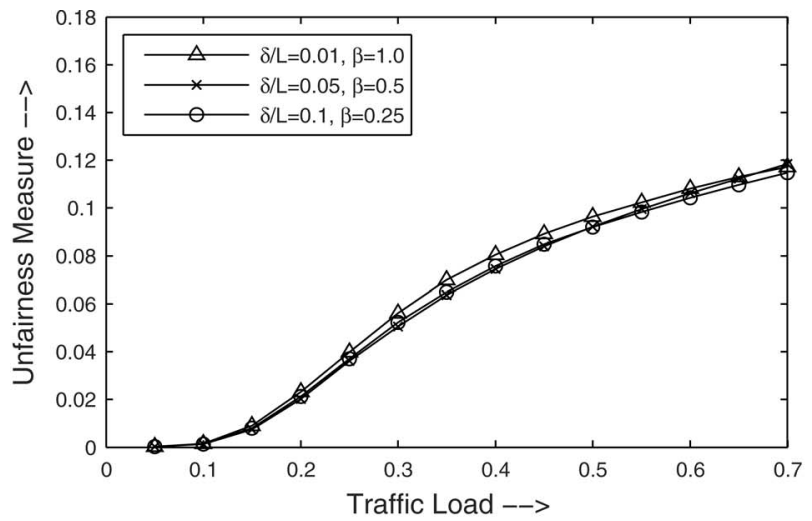

(b)

Fig. 12 Unfairness measure versus traffic load. (a) FPP. (b) CR-FPP. 
ing the bottom in the middle and increasing afterward. Furthermore, the variations on both burst loss and fairness intend to grow larger at higher loads and under more stringent cascading thresholds (i.e., smaller $c_{\max }$ ). We shall explain these two related phenomena as follows. When $\beta$ including negative ones is smaller, bursts with longer hop counts (therefore longer initial offset times) would generally gain more preference during preemption. If $\beta$ is small enough, the overcorrection would occur, resulting in fairness deterioration. If $\beta$ is big enough, bursts with longer hop counts would be suppressed too much, which results in fairness deterioration too. Consequently, fairness reaches the equilibrium state only with a reasonably moderate $\beta$. On the other hand, more longer-hop bursts getting dropped would benefit the other shorter-hop bursts. For example, the resources released because of the discarding of one eighthop burst would probably rescue two or more one-hop bursts. That explains why overall burst loss rates continue to decrease when $\beta$ grows. Certainly, this trend would stop or go inverse if $\beta$ is rather big. Last, at higher loads and under smaller $c_{\max }$, more preemptions are expected to being performed. So the variations brought by different $\beta$ values have occurred faster or appeared larger.

Being able to alleviate the side effect caused by a larger packet processing time $\delta$ is another positive feature through $\beta$ adaptation. In Eq. (2) if $\delta$ becomes bigger but $\beta$ remains unchanged, bursts with longer-hop counts will probably be oversuppressed by the preemption process. This may result in fairness deterioration. Therefore, decreasing $\beta$ shall solve this dilemma. Figures 11 and 12 demonstrate the performance comparisons side by side for CR-FPP and FPP under the effect of different $\delta$ values. The $c_{\max }$ is set to a moderate value of 3 . It is obvious that while FPP suffers from a noticeable performance fluctuation (mostly degradation, especially on the fairness problem) when $\delta$ increases, CR-FPP maintains a very small performance discrepancy by adjusting $\beta$ appropriately.

\section{Conclusion}

In this paper, we reexamine the fairness problem under the effect of the conversion cascading constraint in OBS networks. Both fairness and burst loss performance may deteriorate when bursts, especially those with longer-hop counts, are dropped due to the cascading constraint. Subject to this conversion impediment, we improve an existing preemptive fair channel scheduling algorithm named FPP by integrating the impact of the constraint into the priority evaluation function. The resulting new preemptive scheme CR-FPP also tries to reduce unnecessary wavelength conversions whenever possible. Simulation results show that CR-FPP yields better fairness and achieves lower burst loss rates simultaneously than FPP. It also has the flexibility to work with a diversity of network topologies and to mitigate the side effects resulting from a large packet processing time.

\section{References}

1. J. S. Turner, "Terabit burst switching," J. High-Speed Netw. 8, 3-16 (1999).

2. C. Qiao and M. Yoo, "Optical burst switching (OBS) - a new paradigm for an optical Internet," J. High-Speed Netw. 8, 69-84 (1999).

3. J. Y. Wei and R. I. McFarland, "Just-in-time signaling for WDM optical burst switching networks," J. Lightwave Technol. 18, 20192037 (2000).

4. Y. Xiong, M. Vandenhoute, and H. C. Cankaya, "Control architecture in optical burst-switched, WDM networks," IEEE J. Sel. Areas Commun. 18, 1838-1851 (2000).

5. M. Yoo, C. Qiao, and S. Dixit, "QoS performance of optical burst switching in IP-over-WDM networks," IEEE J. Sel. Areas Commun. 18, 2062-2071 (2000).

6. X. Wang, H. Morikawa, and T. Aoyama, "Deflection routing protocol for burst-switching WDM mesh networks," in IEEE Terabit Optical Networking: Architecture, Control, and Management Issues, Proc. SPIE 4213, 242-252 (2000).

7. K. Inoue and M. Yoshino, "Noise suppression effect in cascaded wavelength conversion using light-injected DFB-LDs," Electron. Lett. 32, 2165-2166 (1996).

8. R. B. Lee, D. F. Geraghty, M. Verdiell, M. Ziari, A. Mathur, and K. J. Vahala, "Cascaded wavelength conversion by four-wave mixing in a strained semiconductor optical amplifier at $10 \mathrm{~Gb} / \mathrm{s}$," IEEE Photonics Technol. Lett. 9, 752-754 (1997).

9. X. Gao, M. A. Bassiouni, and G. Li, "Addressing conversion cascading constraint in OBS networks through proactive routing," Photon. Netw. Commun. 18, 90-104 (2009).

10. C.-F. Hsu and L. C. Yang, "On the fairness improvement of channel scheduling in optical burst-switched networks," Photon. Netw. Commun. 15, 51-66 (2008).

11. J. Xu, C. Qiao, J. Li, and G. Xu, "Efficient channel scheduling algorithms in optical burst switched networks," in Proc. IEEE INFOCOM'03, Vol. 3, pp. 2268-2278 (2003).

12. J. Xu, C. Qiao, J. Li, and G. Xu, "Efficient burst scheduling algorithms in optical burst-switched networks using geometric techniques," IEEE J. Sel. Areas Commun. 22, 1796-1811 (2004).

13. Y. Chen, J. S. Turner, and P.-F. Mo, "Optimal burst scheduling in optical burst switched networks," J. Lightwave Technol. 25, 18831894 (2007).

14. N. Barakat and E. H. Sargent, "Dual-header optical burst switching: a new architecture for WDM burst-switched networks," in Proc. IEEE INFOCOM'05, Vol. 1, pp. 685-693 (2005).

15. N. Barakat and E. H. Sargent, "Separating resource reservations from service requests to improve the performance of optical burstswitching networks," IEEE J. Sel. Areas Commun. 24, 95-107 (2006).

16. C. M. Gauger, "Optimized combination of converter pools and FDL buffers for contention resolution in optical burst switching," Photon. Netw. Commun. 8, 139-148 (2004).

17. N. Akar, E. Karasan, and K. Dogan, "Wavelength converter sharing in asynchronous optical packet/burst switching: an exact blocking analysis for markovian arrivals," IEEE J. Sel. Areas Commun. 24 69-80 (2006).

18. B. Wang and N. Lella, "Dynamic contention resolution in optical burst switched networks with partial wavelength conversion and fiber delay lines," in Proc. IEEE GLOBECOM'04, Vol. 3, pp. 1862-1866 (2004).

19. C.-F. Hsu, T.-L. Liu, and N.-F. Huang, "Performance analysis of deflection routing in optical burst-switched networks," in Proc. IEEE INFOCOM'02, Vol. 1, pp. 66-73 (2002)

20. Y. Chen, H. Wu, D. Xu, and C. Qiao, "Performance analysis of optical burst switched node with deflection routing," in Proc. IEEE Int Conf on Communications (ICC'03), Vol. 2, pp. 1355-1359 (2003).

21. S.-K. Lee, K. Sriram, H.-S. Kim, and J.-S. Song, "Contention-based limited deflection routing in OBS networks," in Proc. IEEE GLOBECOM'03, Vol. 5, pp. 2633-2637 (2003).

22. A. Zalesky, H. L. Vu, M. Zukerman, Z. Rosberg, and E. W. M. Wong, "Evaluation of limited wavelength conversion and deflection routing as methods to reduce blocking probability in optical burst switched networks," in Proc. IEEE Int. Conf. on Communications (ICC'04), Vol. 3, pp. 1543-1547 (2004).

23. V. A. Vokkarane, J. P. Jue, and S. Sitaraman, "Burst segmentation: an approach for reducing packet loss in optical burst switched networks," in Proc. IEEE Int. Conf. on Communications (ICC'02), Vol. 5, pp. 2673-2677 (2002)

24. Z. Rosberg, H. L. Vu, M. Zukerman, and J. White, "Performance analyses of optical burst-switching networks," IEEE J. Sel. Areas Commun. 21, 1187-1197 (2003).

25. H. C. Cankaya, S. Charcranoon, and T. S. El-Bawab, "A preemptive scheduling technique for OBS networks with service differentiation," in Proc. IEEE GLOBECOM'03, Vol. 5, pp. 2704-2708 (2003).

26. T. Tachibana, M. Ueda, and S. Kasahara, "A preemptive scheme with two-way release message transmission in optical burst switching networks," in Proc. IEEE GLOBECOM'04, Vol. 3, pp. 1994-1998 (2004)

27. E. Magana, D. Morato, M. Izal, and J. Aracil, "Evaluation of preemption probabilities in OBS networks with burst segmentation," in Proc. IEEE Int. Conf. on Communications (ICC'05), Vol. 3, pp. 1646-1650 (2005).

28. H. Li, M. Tan Wei Liak, and I. Thng Li-Jin, "A distributed 
monitoring-based fairness algorithm in optical burst switching networks," in Proc. IEEE Int. Conf. on Communications (ICC'04), Vol. 3, pp. $1564-1568$ (2004)

29. B. Zhou, M. A. Bassiouni, and G. Li, "Improving fairness in opticalburst-switching networks," J. Opt. Netw. 3, 214-228 (2004).

30. B. Zhou, M. A. Bassiouni, and G. Li, "Using constrained preemption to improve dropping fairness in optical burst switching networks," Telecommun. Syst. 34, 181-194 (2007)

31. X. Gao and M. A. Bassiouni, "Improving fairness with novel adaptive routing in optical burst-switched networks," J. Lightwave Technol. 27, 4480-4492 (2009).

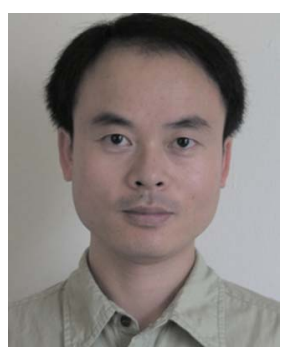

Xingbo Gao received his $\mathrm{MSc}$ and $\mathrm{PhD}$ degrees in computer science from the University of Central Florida, Orlando, in 2006 and 2009, respectively. His research interests include optical networks, network modeling, and network security, with an emphasis on optical networking.

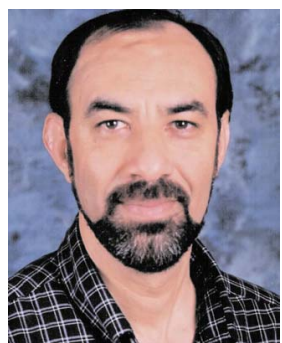

Mostafa A. Bassiouni received his BSc and MSc degrees in computer science from Alexandria University and received his $\mathrm{PhD}$ degree in computer science from Pennsylvania State University in 1982 . He is currently a professor of computer science at the University of Central Florida, Orlando. His research interests include distributed systems, computer networks, real-time protocols, and concurrency control. He has authored over 175 papers published in various computer journals, book chapters, and conference proceedings. His research has been supported by grants from ARO, ARPA, NSF, STRICOM, PM-TRADE, CBIS, Harris, and the State of Florida. He has served as member of the program committee of several conferences, as the program committee chair of CSMA '98 and CSMA '2000, and as the guest co-editor of a special issue of the Journal of Simulation Practice and Theory, 2002. He is also serving as an editorial board member of the Computer Journal, Oxford University Press.

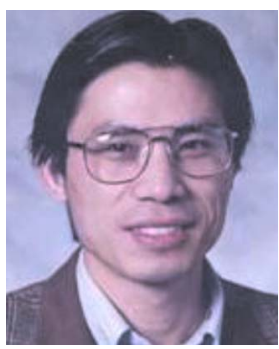

Guifang $\mathbf{L i}$ is a full professor in the College of Optics and Photonics at the University of Central Florida. He received his $\mathrm{PhD}$ degree in electrical engineering from the University of Wisconsin at Madison. His research interests include all-optical signal processing for optical networks, fiber-radio systems, RF photonics, and nonlinear dynamics. He is the recipient of the NSF

Presidential Young Investigator award and the Office of Naval Research Young Investigator award. He is currently director of the NSF IGERT program in optical communications and networking. He was an associate editor of Optical Networks (1999 to 2003). 